\title{
Confucianismo y democracia: ciudadanos, príncipes, individuos ${ }^{1}$ Confucianism and Democracy: Citizens, Princes, Individuals
} Aurelio de Prada García ${ }^{2}$

Universidad Rey Juan Carlos

"Toda persona tiene derecho a participar en el gobierno de su país,
directamente o por medio de representantes libremente elegidos."
Declaración Universal de los Derechos Humanos. Art. 21.1
“... $\sin$ salir de su casa puede tener influencia en el reino"
La Gran Enseñanza, IX

RESUMEN: En este artículo se aborda la cuestión de si la democracia es o no compatible con la perspectiva que, según opinión generalizada, constituye la base de la cultura china: el confucianismo. Una perspectiva que es analizada tanto desde su marco contextual como en sí misma, cotejándola con el concepto de democracia. El resultado de todo ello lleva a la conclusión de que la democracia es ajena al confucianismo y por ello a la cultura china. Sin embargo, la democracia no es necesariamente incompatible con tal perspectiva hasta el punto de que cabria imaginar una síntesis entre ambas.

Palabras clave: Ciudadanos, Confucianismo, Democracia, Individuos, Príncipes, Idiotés.
ABSTRACT: In this article we focus on the question of whether democracy is compatible with the point of view that, according to general opinion, is at the very core of Chinese culture: Confucianism. This point of view is analysed in its contextual frame as well as within itself comparing it with the concept of democracy. The conclusion is that democracy is foreign to Confucianism and, by extension, to Chinese culture. Ultimately, however, democracy is not necessarily incompatible with that perspective. It is possible to imagine a synthesis between them.

Key words: Citizens, Confucianism, Democracy, Individuals, Princes, Idiotés. 


\section{Introducción}

No es preciso extenderse demasiado para mostrar el ascenso que viene experimentando China en los últimos decenios. Basta citar el dato de que su economía está a punto de sobrepasar a la de EEUU, convirtiéndose en la primera economía del mundo y acabando, de paso, con una hegemonía occidental que ha durado siglos. Así las cosas, resulta más que oportuno examinar, con cierto detenimiento, si su cultura es o no compatible con una "invención"3 que impregna tan marcadamente la civilización occidental, — hasta casi definirla-, como es la de la democracia: la participación en el gobierno del propio país, directamente o por medio de representantes libremente elegidos, tal y como se recoge al principio de estas líneas.

\section{Problemas de método}

Desde luego no parece haber mayor problema en averiguar cuáles serían los rasgos definitorios de esa cultura ascendente: los del confucianismo. ${ }^{4}$ En efecto, hay acuerdo generalizado en que si de China quitásemos el confucianismo, su historia y su cultura se harían tan incomprensibles como las de Europa sin filosofía griega y sin cristianismo ${ }^{5}$ y es que el "fundador", _ por así decirlo-, de esa perspectiva, Confucio, más que un hombre o un pensador, habría sido un auténtico fenómeno cultural que se confundiría con el destino de toda la civilización china. ${ }^{6}$ Una civilización sobre la que habría tenido una influencia comparable a las de Sócrates y Cristo combinadas en Occidente, ${ }^{7}$ pudiéndose afirmar en consecuencia que China no sería sino una "creación de Confucio". 8
Sí hemos de atender, sin embargo, una cuestión metodológica que obliga a analizar esos rasgos tal y como aparecen expresados en chino y no en castellano algo que puede verse inmediatamente a partir de los propios caracteres chinos que se vierten al castellano como "confucianismo" o "escuela de los letrados": 儒 家, rú jiā.

Ciertamente podemos traducir sin mayores problemas por "escuela" el segundo carácter, 家, jiā/casa, pues es una composición de 豕 shı̌/cerdo (se pueden imaginar fácilmente la cabeza, las patas y la cola) debajo de ウ băogaì / cobertizo, techo: el cerdo bajo el techo, o sea, la casa, el hogar, ${ }^{9}-$ los que están reunidos bajo el cerdo colgado del techo- y desde ahí, "escuela", —los que se reúnen alrededor de un maestro-, pero no resulta tan fácil traducir el primero de esos caracteres.

Literalmente habría que traducir 儒 rú como "hombre que hace que haya lluvia para las plantas que acaban de brotar de la tierra"10 dado que se compone de otros tres: 1 rén/ hombre, 雨 yǔ /lluvia (se pueden ver las nubes, las gotas de agua y un rayo) sobre el carácter 而 er/planta recién brotada de la tierra ( es fácil imaginar las raíces, el suelo y la planta sobresaliendo), de modo que cabría traducirlo como "el hombre" o mejor, ya que se trata de una escuela, 家 "los hombres necesarios para que haya lluvia para las plantas recién brotadas".

Así las cosas, si tradujéramos 儒家 rú jīa por "confucianismo", estaríamos tergiversando por completo la expresión dado que, en chino, el nombre de Confucio no figura en la denominación de "su" escuela, y si nos quedáramos con la traducción "escuela de letrados", perderíamos todo el universo semántico que según hemos visto incorpora la 
unión de "hombre", "lluvia" y "planta recién brotada" en un solo carácter.

En conclusión, a la hora de examinar el "confucianismo", — término que seguiremos utilizando por comodidad y por su uso generalizado, si bien ha de entenderse en adelante que incorpora los significados que acabamos de señalar-, por un mínimo rigor metodológico, resulta obligado analizar cada uno de los caracteres en los que se expresa ese modo de estar en el mundo, para recoger en la medida de lo posible su universo semántico y poder cotejarlo así con la gran invención occidental de la democracia.

Algo que, por cierto, ya podemos hacer, pues de lo anterior parece seguirse que no hay compatibilidad alguna entre democracia y confucianismo. En efecto, "democracia" significa literalmente el poder, cratos, del pueblo, demos, algo que parece completamente ajeno al confucianismo; a los "hombres necesarios para que haya lluvia para las plantas recién brotadas" a los que acabamos de llegar.

Ciertamente, podría forzarse la argumentación aduciendo que sí habría compatibilidad entre "democracia" y "confucianismo" pues, en ambos casos, de un modo u otro, se habla de "poder". Un "poder" abstracto en el caso de la democracia, y un "poder" concreto, —el de invocar la lluvia para las plantas recién brotadas-, en el caso del "confucianismo". No parece preciso, sin embargo, abundar en lo forzado de semejante argumentación.

En efecto, literalmente la definición de democracia enfatiza, por así decirlo, el sujeto del poder: el pueblo. Un sujeto que, por lo demás no se especifica; no se indica a quien incluye ${ }^{11}$ sino que aparece simplemente como titular de ese poder en abstracto. Así las cosas, resulta evidente que la democracia juega en un universo semántico en el que aparece como una alternativa entre otras posibles. El pueblo, demos, aparece como un titular del poder entre otros posibles: monarquía, oligarquía, tiranía... tal y como puede corroborarse a lo largo de toda la tradición occidental. ${ }^{12}$

Por el contrario, en el confucianismo el énfasis se pone en el objeto de ese poder, o mejor, de esa capacidad concreta de invocar la lluvia. Énfasis que permite distinguir a los sujetos que poseen tal capacidad frente a los que no; sin que haya por tanto diversas alternativas como ocurre en el universo semántico en el que aparece la democracia. La capacidad de hacer que llueva, de invocar la lluvia se tiene o no se tiene; tertium non datur.

Por lo demás, en el confucianismo, en la escuela de los rú 儒家, se da una conexión entre naturaleza y sociedad que no aparece en el universo semántico en el que juega la democracia. En efecto, los rú 儒 son hombres "necesarios" natural y socialmente, si es que cabe hablar así, en términos claros y distintos. Naturalmente necesarios para que haya lluvia para las plantas recién brotadas y socialmente necesarios por lo mismo, ya que las plantas son necesarias para la supervivencia de la sociedad.

Por el contrario y pese a que la conexión naturaleza sociedad está presente asimismo en la tradición occidental, — pudiendo rastrearse, sin ir más lejos, en la dualidad derecho natural y derecho positivo-, lo cierto es que no aparece en absoluto en la definición de democracia. Una definición de la que se sigue simplemente que el poder reside en el pueblo como una mera posibilidad frente a otras. Así las cosas, parece obliga- 
do concluir que confucianismo y democracia serían incompatibles al jugar en dos universos semánticos completamente distintos.

Ahora bien esta conclusión resulta prematura, pues hemos llegado a ella desde el análisis de las meras denominaciones "confucianismo" y "democracia" con lo cual, ciertamente, procede dejarla en suspenso y pasar a examinar en detalle todo el discurso que comporta el confucianismo cotejándolo con la gran invención occidental de la democracia para acabar de validar dicha conclusión o, en su caso, llegar a una diferente.

\section{Fieles y ciudadanos}

"Confucianismo" venimos diciendo y no del todo bien, pues, - al margen del universo semántico que, como acabamos de ver, incorpora la expresión-, no hemos señalado que Confucio se consideraba un mero amante de los antiguos ${ }^{13} \mathrm{y}$ que su enseñanza se inscribe en una visión previa del mundo. ${ }^{14}$ Un marco previo que hemos de analizar, pues, en primer término. Algo que no resulta excesivamente complejo pues dicho marco figura aún hoy en los templos confucianos: $:^{15}$

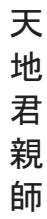

Estos caracteres pueden traducirse como "cielo, tierra, rey, padres, maestros". ${ }^{16}$ Traducción que, —según las consideraciones metodológicas hechas más arriba-, no podríamos aceptar sin antes examinar el universo semántico que cada uno de esos caracteres incorpora. Algo que ya hemos hecho en otro lugar al que nos remitimos ${ }^{17} \mathrm{si}$ bien hemos de hacer hincapié en el carácter central de esa serie pues parece obligarnos a matizar la incompatibilidad entre democracia y confucianismo a que acabamos de llegar.

En efecto, en el centro de la serie de caracteres cuya traducción acaba de validarse aparece el monarca, el rey, 君 jün, 一representado como la boca, 口 kóu del que empuña el cetro con su mano尹, yin/ como se ve casi inmediatamente. Con todo lo cual se sigue que el marco conceptual en el que se mueve el confucianismo incluye la presencia de una de las alternativas que, como vimos, aparecen en el universo semántico en el que se incluye la democracia.

Así las cosas, si bien habría de concluirse que democracia y confucianismo son incompatibles, - en cuanto que democracia y monarquía lo son-, al menos el universo semántico en que se mueven seria el mismo. La monarquía se presentaría como una alternativa posible a la democracia y viceversa, al igual que en la tradición occidental.

Ahora bien, sólo lo parece, pues "la boca del que empuña el cetro", - el monarca, 君一, no se presenta sin más, en abstracto, como mera alternativa a la democracia, al poder del pueblo también en abstracto, sino que juega en un contexto concreto, perfectamente delimitado por el resto de los caracteres de la serie. Unos caracteres que, por cierto, no son elementos previos, - "claros y distintos", por decirlo con Descartes ${ }^{18}$ 一, que entran en relación entre sí sino que, por el contrario, se constituyen como tales en su relación, generando al tiempo la secuencia misma en la que se incluyen. ${ }^{19}$

Más aún, la secuencia es dinámica, regenerándose continuamente, con lo cual hay un punto más importante que los demás: el 
central, el que permite el dinamismo constante de la secuencia. ${ }^{20}$ Algo que se sigue de la propia lengua china y es que, en efecto, 中 zhōng/ medio, "centro" no es un término sólo nominal sino también verbal; no sólo designa la centralidad espacial que se ocupa sino la virtud dinámica y activa que corresponde a ese lugar: la de la flecha en el centro del blanco. ${ }^{21}$

Un punto más importante decimos y no decimos del todo bien, pues se podría afirmar sin mayores problemas que el punto central,- - la centralidad así entendida- - define a la propia civilización china y es que, como es bien sabido, China en chino es 中国, zhōng guó: el país, 国 guó del centro, 中 zhōng; el país situado en el centro y con la virtud correspondiente.

Así las cosas, en la serie天 地君 親師, “cielo, tierra, rey, padres, maestros" cabría ver dos mundos: 1) el natural, —-formado por el cielo, 天 y la tierra, 地一, y 2) el socialcultural, - formado por la familia extensa 親 y los maestros 師一. Dos mundos integrados por el punto central, por el rey, 君. el que sostiene con su mano el cetro, 尹, inmediatamente debajo del cielo y la tierra, 天地 mientras que con su boca, 口, da órdenes, organiza, integrándolo en el continuo, el mundo social, 親 師.

Desde luego, no resultan ajenos a la tradición europea ni la correspondencia entre naturaleza y sociedad, como se apuntó más arriba, ${ }^{22}$ ni el modo en que se corresponden por medio del rey, del monarca, ${ }^{23}$ sino todo lo contrario. Asimismo tampoco resulta extraña a la tradición política occidental la idea de centro entendida como la flecha que da en el blanco como ya aparece en Aristóteles:

"Si existe, pues, algún fin de nuestros actos que queramos por él mismo y los demás por él, y no elegimos todo por otra cosa - pues así se seguiría hasta el infinito, de suerte que nuestro deseo sería vacío y vano- es evidente que ese fin será lo bueno y lo mejor. Y así, ¿no tendrá su conocimiento gran influencia sobre nuestra vida y, como arqueros que tiene un blanco, no alcanzaremos mejor el nuestro? Si es así, hemos de intentar comprender de un modo general cuál es y a cuál de las ciencias o facultades pertenece: Parecería que ha de ser el de la más principal y eminentemente directiva. Tal es manifiestamente la política." 24

Ahora bien, todo ello no ayuda a la compatibilidad sobre la que indagamos, pues mientras en el confucianismo la monarquía aparece dentro de un marco, - como el centro de una secuencia (天 地君 親 師 "cielo, tierra, rey, padres, maestros") que incluye la naturaleza y la sociedad garantizando la armonía entre ambas - , la democracia aparece completamente descontextualizada. Es el poder del pueblo, a secas, sin referencia alguna al ámbito en el que ese poder se ejerce, - natural o social-, e incluso sin referencia al propio significado del término "pueblo".

Ciertamente y de nuevo, se podría intentar forzar la argumentación para hacer compatibles confucianismo y democracia aduciendo, por ejemplo, que la idea de centro sí que está presente en la democracia, pues lo que viene a enfatizarse con esa denominación es precisamente la centralidad del pueblo en la acción política, por así decirlo. Ahora bien, no parece preciso insistir en lo artificioso de esa argumentación, pues esa centralidad apenas si puede parangonarse con el sentido que "centro" tiene en chino y que acaba de ilustrarse. Así las cosas, hemos de concluir que democracia y confucianismo resultan incompatibles, no sólo desde los universos semánticos in- 
cluidos en las meras denominaciones sino también en relación al marco de pensamiento en que se mueve el confucianismo.

Pero esta conclusión resulta una vez más precipitada y es que lo anterior no agota el análisis del marco en que se mueve el confucianismo. Ciertamente, hasta aquí hemos procedido a dicho análisis desde un punto de vista puramente externo ${ }^{25}$ obviando el hecho de que la secuencia 天地君親師 “cielo, tierra, rey, padres, maestros" es algo vivo, algo presente a los ojos de sus adeptos, de sus fieles, 忠. Más aún, no se trata sólo de una mera presencia viva sino de una que incluye, que religa, vinculando literalmente en la secuencia, ${ }^{26}$ al fiel, al 忠 zhōng: el que tiene el centro en medio del corazón.

En efecto, el carácter 忠, “adepto”, “fiel” está compuesto por otros dos. El de arriba 中 zhōng "centro", ya lo conocemos; ${ }^{27}$ un carácter a la vez nominal y verbal, el centro- el medio y la virtud que conlleva esa posición: la de la flecha en el centro del blanco. El otro, debajo, es el carácter 心 xīn “corazón", un pictograma en el que se ven tres gotas de sangre deslizándose por los ventrículos. Con todo lo cual el fiel, el 忠 zhōng, es efectivamente el que lleva el centro en medio del corazón o, si se quiere y mejor, el que tiene su corazón en el centro. Así las cosas, ya podemos completar nuestro análisis del marco previo en el que se mueve el confucianismo incluyendo en él también al fiel, esto es:

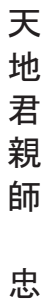

Ni que decir tiene, que esta visualización no es ajena a la tradición occidental ya que se compadece, mutatis mutandis, con la de un miembro de la polis, un ciudadano, un polites, sintiendo que "la ciudad es por naturaleza anterior a... cada uno de nosotros", por decirlo con Aristóteles ${ }^{28}$ y también, mutatis mutandis, con la tradición iusnaturalista clásica en su versión estoica; con el perro que va atado a la parte trasera de un carro en movimiento. Perro que no es sino el hombre, mientras que el carro simboliza el destino, la razón universal que, como ley eterna, determina el criterio del comportamiento al que debe ajustarse el perro. Si es inteligente, lo que hace es seguir dócilmente al carro; si se resiste, apoyándose en las patas de atrás, lo único que logra es ser arrastrado:

\section{Fata volentem ducunt, nolentem trahunt ${ }^{29}$.}

Ahora bien, esa visualización resulta completamente ajena a la idea de democracia, —al menos, en la concepción modernadonde ya no cabe hablar de un fiel, de un ciudadano, de un polites o de un perro que aparecen como meras partes de un todo anterior a ellos, sino de "individuos", de elementos previos aislados entre sí, — dignos en sí mismos, con "derechos humanos"-, que constituyen el todo, el edificio social en el que se integran: el "pueblo". Algo que se muestra, sin ir más lejos, en uno de los mayores teóricos de la democracia: J. J. Rousseau, para quien el contrato social es precisamente "el acto en virtud del cual un pueblo es un pueblo". ${ }^{30}$

Ciertamente no procede abundar en la incompatibilidad entre confucianismo y democracia a la que de nuevo hemos llegado. Mucho menos procede abundar en la ruptura dentro de la propia tradición occidental que 
parece seguirse de lo anterior y según la cual habría dos tipos de ciudadanos, los antiguos y los modernos, ${ }^{31}$ correspondientes a dos concepciones de la democracia y es que, hasta aquí sólo hemos analizado el marco en que se inscribe el confucianismo, pero no el confucianismo propiamente dicho. Con todo lo cual, una vez más, hemos de considerar provisional lo anterior y pasar a analizar las aportaciones que el confucianismo hace a ese marco para acabar de validar, o no, su incompatibilidad con la democracia.

\section{Príncipes e individuos}

Aportaciones que podemos identificar inmediatamente desde uno de los "Cuatro libros" del "canon confuciano": 32 las Analectas cuyo tema central es definir las cualidades que corresponden al 君子 jūnž̌, una expresión no inventada por Confucio pues ya aparece en textos anteriores designando a cualquier miembro de la nobleza. ${ }^{33}$ Una vez más, sin embargo, no podemos conformarnos con esa traducción sino que, - cumpliendo con nuestros presupuestos metodológicos - , hemos de examinar el universo semántico de esos caracteres.

El primero de ellos, 君 jün /monarca, ya lo conocemos dado que es el que ocupa el centro de la serie 天地君 親師, “cielo, tierra, rey, padres, maestros" permitiendo la interrelación y el dinamismo de toda la secuencia. En cuanto al segundo, 子 ž̆, puede traducirse sin mayores problemas por "hijo", - hijo varón- ya que, como se aprecia casi inmediatamente, es el pictograma de un niño pequeño, un bebé con los brazos extendidos y envuelto en pañales. Así las cosas, 君子 significa “el hijo del rey”, "el príncipe", y desde ahí, por extensión, "noble”, cualquier miembro de la nobleza.
Ahora bien, el mero examen del universo semántico incorporado en esos caracteres no permite aprehender por completo el nuevo sentido que Confucio, - aun manteniéndolo-, da al término: cualquiera, con independencia del nacimiento, puede convertirse en 君子, en hijo del rey, en príncipe, y ello por medio de la educación adecuada. ${ }^{34}$

Un nuevo sentido que se sigue plenamente atendiendo al contexto histórico en que Confucio lo formula: el tiempo inmediatamente anterior al caos de la época de los "Reinos Combatientes”, 战国 (463-221 a. C.). Un tiempo en el que ya no había un rey, un 君 que asegurase, - desde el centro de la secuencia, 天地君 親師 “cielo, tierra, rey, padres, maestros" —, la armonía natural-social sino varios hijos del rey, varios 君子, peleando entre sí en un estado de guerra de todos contra todos. ${ }^{35}$ La solución que da Confucio a esa quiebra del centro consiste en lo que bien podría llamarse una democratización de la monarquía. Consiste en trasladar el poder, el centro, a los adeptos, a los fieles a los que ahora, por medio de la educación apropiada, se les hace sustentadores de la armonía, literalmente hijos del rey, 君子 “príncipes”.

Ya tenemos, pues, una caracterización completa del confucianismo que, según se ve, mantiene el marco en el que se inscribía con la única modificación de convertir, por medio de la educación apropiada, al fiel en príncipe:

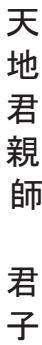


Una modificación mínima pero capital. Ahora, el fiel 忠 convenientemente educado, convertido en príncipe 君子, pasa a ocupar el centro. Ahora, "sin salir de su casa puede tener influencia en el reino"36, puede contribuir a la armonía de toda la secuencia. Una armonía natural-social como vimos en su momento y como puede corroborarse también desde otro de los "Cuatro libros" confucianos: 中 庸 zhōng yōng, la Doctrina del Centro, cuyo tema principal es, precisamente, la interrelación, la "unidad" del mundo natural y social. ${ }^{37}$ Ahora el fiel 忠 se convierte en $r u$ 儒, en el hombre natural y socialmente necesario; el que hace que haya lluvia para las plantas necesarias para la supervivencia de la sociedad.

Una "democratización de la monarquía" decíamos y, sin embargo, decíamos mal, pues de lo anterior se sigue, más bien y de nuevo, la incompatibilidad entre democracia y confucianismo. En efecto, frente a ese centro extendido a todos los fieles educados del confucianismo; frente a esa acción armonizadora que puede ejercerse desde la propia casa, se alza la democracia como mera afirmación del poder abstracto de "individuos", valiosos en sí mismos, con independencia de cualquier consideración natural o social, que se constituyen en pueblo sin necesidad de educación específica alguna. ${ }^{38}$

Un poder que, por lo demás, se suele delegar en representantes bien porque no se quiera, bien porque no se pueda ejercerlo. Así, frente a los "príncipes por educación" del confucianismo estaríamos los "idiotas" de las democracias modernas. "Idiotas" en los dos sentidos del término idiotes. Primero, en el de "persona particular" que no quiere ejercer los derechos políticos y prefiere dedicarse a sus propios asuntos:
"Resígnese, pues, el poder: lo que nosotros necesitamos es la libertad, la cual conseguiremos indefectiblemente; pero como la que necesitamos es diferente de la de los antiguos, es necesario que se dé a aquélla una organización diferente a la que podría convenir a la libertad de los antiguos; en ésta, el hombre cuanto más consagraba el tiempo y su fuerza para el ejercicio de los derechos políticos, más libre se creía; por el contrario, en la especie de libertad de que nosotros somos susceptibles, cuanto más tiempo nos deje para nuestros intereses privados el ejercicio de los derechos políticos, más preciosa será para nosotros la misma libertad.

De aquí viene la necesidad del sistema representativo, el cual no es otra cosa que una organización con cuyo auxilio una nación se descarga sobre algunos individuos de aquello que no quiere o no puede hacer por sí misma.”39

Después en el de "persona ignorante", "ruda", inexperta... que no sólo renuncia ${ }^{40}$ a ejercer sus derechos políticos, prefiriendo que sus representantes actúen por él, sino que se reconoce, más o menos explícitamente, inferior a ellos, - "idiota"-, como puede seguirse, sin ir más lejos, de la vigente regulación constitucional de la iniciativa legislativa popular ${ }^{41} \mathrm{y}$ del referéndum para decisiones políticas de especial trascendencia. ${ }^{42}$

Con todo lo cual, ciertamente resulta obligado concluir que democracia y confucianismo son incompatibles no sólo desde las meras denominaciones o desde el marco en que se mueve el confucianismo, sino también desde las aportaciones que hace Confucio a dicho marco. Incompatibilidades que bien pueden resumirse en la contraposición entre "príncipes" que son partes de un todo que les antecede y a cuya armonía pueden contribuir incluso desde su casa e "indivi- 
duos" valiosos por sí mismos, con independencia de cualquier todo natural o social, que prefieren convertirse en "idiotas" dejando a sus representantes ejercer el poder por ellos y reconociéndose inferiores a tales representantes.

\section{A modo de conclusión: realizar la democracia}

Ahora bien y una vez más esta conclusión es provisional, pues, ciertamente, cabría imaginar una síntesis entre confucianismo y democracia, entre príncipes e individuos. Más aún, no sólo cabría sino que parece imprescindible en un momento en que la "casa" común, el planeta Tierra, está perdiendo las condiciones que permiten la supervivencia del género humano, con lo cual resulta más que urgente una actuación que ponga freno al cambio climático y contribuya a que haya la lluvia necesaria para las plantas recién brotadas 儒. ${ }^{43}$

Para ello, ciertamente, los individuos de las democracias contemporáneas deberíamos renunciar a nuestra "idiotez" y pasar, por medio de la educación apropiada, a reconocernos partes de un todo, convirtiéndonos así en príncipes que, incluso desde la propia casa, desde el propio oikos, aseguran la armonía de ese todo previo a nosotros. Unos príncipes que, por supuesto, no renegarían sino todo lo contrario del valor intrínseco, de la dignidad del ser humano individual con independencia de su pertenencia a un todo previo; no renegarían de los derechos humanos. Explicitar los rasgos de esa "realización de la democracia", de ese hacerla real en el sentido de "auténtica" y también en el de "realeza" es algo que, sin embargo, ciertamente supera los límites asignados a estas líneas. ${ }^{44}$

\section{BIBLIOGRAFIA}

Arblaster, A.: Democracia. Alianza, Madrid 1992.

Aristoteles, Política, Instituto de Estudios Políticos, Madrid 1970

Aristoteles, Ética a Nicómaco, Centro de Estudios Constitucionales, Madrid 1976.

Ceinos, P.: Manual de Escritura de los Caracteres Chinos, Miraguano, Madrid 2006.

Cheng, A.: Historia del pensamiento chino. Bellaterra, Barcelona, 2002.

Cheng, F.: La escritura poética china. Pretextos, Valencia, 2007.

Confucius: The Analects. Chinese-English edition, Taipei. 2006.

Constant, B. De la libertad de los antiguos comparada con la de los modernos. Tecnos, Madrid 1988.

De Mente, B.: The Chinese have a word for it: the complete guide to Chinese thought and culture." NTC, Pub. Group, Chicago 2000.

Descartes: Discurso del método, Ed. Alfaguara, Madrid, 1981

Eliade, M.: Historia de las creencias y las ideas religiosas. Vol. II. Ediciones Cristiandad. Madrid, 1979.

Folch, D.: La construcción de China. El período formativo de la civilización china, Península/Atalaya, Barcelona 2001.

Hart, H. La.: El concepto de Derecho, Editora Nacional, México 1980.

Hesiodo: Trabajos y días, en Obras y Fragmentos, Gredos, Madrid, 2006.

Hunt, L: La invención de los derechos humanos, Tusquets, Barcelona 2009 
Kelsen, H.: La idea del Derecho Natural y otros ensayos. Editora Nacional. México, 1979.

Li, L.: Tracing the roots of Chinese Characters: 500 cases. Beijing Language and Culture University Press, Beijing 1993.

McNaughton. W. y Ying, L.: Reading \& Writing Chinese. Traditional Character Edition. Tuttle Publishing, Singapore 1999.

Oldstone Moore, J.: "El confucianismo: Orden y virtud.” en Shaughnessy, E. L. (Ed.): China, Blume, Barcelona 2008.

Perez Arroyo, J.: "Introducción” en Confucio: Los cuatro libros. RBA, Barcelona 2006.

Rousseau, J.J.: El contrato social, en Escritos de Combate, Alfaguara, Madrid 1978.

Smith; H.: Las religiones del mundo. Círculo de Lectores, Barcelona 2001, p. 189

Sofocles: Tragedias completas. Gredos, Madrid 2010,

Truyol y Serra, A.: Historia de la Filosofia del Derecho y del Estado. Vol. 1 Alianza, Madrid 1978.

Ullmann, W.: Principios de gobierno y política en la Edad Media, Madrid, Alianza Universidad, 1985.-

Van Norden, B.W.: "Introduction" en Van Norden, B.W. (Ed): Confucius and the Analects. New Essays, Oxford University Press, Oxford 2002,

Welzel, H. Introducción a la Filosofía del Derecho, Aguilar, Madrid 1979.

Xingzhong, Y.: El confucianismo, Cambridge University Press, Madrid 2001.

\section{NOTAS}

${ }^{1}$ Este trabajo se inscribe dentro del proyecto de investigación "Principio de no discriminación y nuevos derechos". (DER2011-26903)

${ }^{2}$ Doctor en Derecho por la UCM. Profesor en la UCM, UB y actualmente Profesor Titular de Filosofía del Derecho en la URJC, habiendo sido asimismo Profesor Tutor en la UNED. Estancias de investigación en la Universidad de Coimbra; en la National Taipei University y en La Sapienza. Entre sus publicaciones destacan: De toute la force commune, Editorial de la Universidad Complutense, Madrid 1989, _-sobre la filosofía política de J.J. Rousseau - , e Individuos $y$ Reyes, Servicio Publicaciones URJC, Madrid 2011, - una síntesis entre confucianismo y derechos humanos - . Ha publicado asimismo numerosos artículos y capítulos de libro sobre cuestiones de legitimidad democrática, ecologismo, globalización y glocalización, derechos humanos, confucianismo, budismo y metodología docente de la Teoría y la Filosofía del Derecho. También ha realizado traducciones de textos en alemán, italiano y portugués. aurelio.deprada@urjc.es.-
${ }^{3}$ Parafraseando el título con el que se ha traducido la obra de Hunt, L: Inventing Human Rights. Vid. La invención de los derechos humanos. Tusquets, Barcelona 2009.

${ }^{4}$ Paradójicamente, el confucianismo habría contribuido, incluso a consolidar al propio régimen comunista vigente .Vid. De Mente, B.: The Chinese have a word for it: the complete guide to Chinese thought and culture." NTC, Pub. Group, Chicago 2000, p. 28: "The similarities between Chinese traditionalism and Communist ideology is no doubt one of the reasons why communism, in its firsts decades, was as successful as it was in China."

${ }^{5}$ Pérez Arroyo, J.: "Introducción" en Confucio: Los cuatro libros. RBA, Barcelona 2006, p. 7.

${ }^{6}$ Cheng, A.: Historia del pensamiento chino. Bellaterra, Barcelona, 2002 p. 55 y 56.

7 "Imagine a person who has an influence on his native tradition comparable to the combined influence of Jesus and Socrates on the Western tradition. Such a person was Confucius." Van Norden, B.W.: "Introduction" en Van Norden, B.W. 
(Ed).: Confucius and the Analects. New Essays, Oxford University Press, Oxford 2002, p 3.

${ }^{8}$ Truyol y Serra, A.: Historia de la Filosofía del Derecho y del Estado. Vol. 1 Alianza, Madrid 1978. p. 74.

${ }^{9}$ Para las traducciones de los caracteres chinos hemos utilizado: Ceinos, P.: Manual de Escritura de los Caracteres Chinos, Miraguano, Madrid 2006, LI, L.: Tracing the roots of Chinese Characters: 500 cases. Beijing Language and Culture University Press, Beijing 1993 y McNaughton. W. y Ying, L.: Reading \& Writing Chinese. Traditional Character Edition. Tuttle Publishing, Singapore 1999.

${ }^{10}$ Sobre la relación de los rú 儒 con la danza ritual de oración por la lluvia vid. Xingzhong, Y.: El confucianismo, Cambridge University Press, Madrid 2001. p. 41.

${ }^{11}$ Arblaster, A.: Democracia. Alianza , Madrid 1992 p. 19

12 Vid., por todos, Aristóteles, Política, $1278 b$ y ss.

${ }^{13}$ Confucius: The Analects. Chinese-English edition, VII, 1 Taipei 2006.

${ }^{14}$ Folch, D.: La construcción de China. El período formativo de la civilización china, Barcelona, Península/Atalaya, 2001. p. 138.

${ }^{15}$ Cheng, F.: La escritura poética china. Pretextos, Valencia, 2007, p. 29.

${ }^{16}$ Ibid.

${ }^{17} \mathrm{He}$ analizado esa secuencia de caracteres en las siguientes publicaciones: Entre confucianismo y derechos humanos: 君人 Individuo y rey. Cuadernos electrónicos de Filosofía del Derecho, n. 23, junio de 2011, p 131-159, Between Confucianism and Human Rights: 君 人 Individuals and Kings. Rechtstheorie, Duncker \& Humblot, Berlin 2012, Vol. 43, No. 2 pp. 221239 y con mayor detalle en Individuos y reyes. Servicio de Publicaciones de la URJC, Madrid 2011.

${ }^{18}$ Discurso del método, Ed. Alfaguara, Madrid, 1981, pág. 15.

${ }^{19}$ Para el pensamiento chino, el concepto de relación no es un simple lazo que se establece entre entidades antes distintas, sino algo que las constituye como tales; es "constitutiva de los seres en su existencia y devenir". Cheng, A.: Historia del pensamiento chino. cit., p. 37
20 “中 zhōng " medio", "centro", no es un término sólo nominal sino también verbal; no sólo designa la centralidad espacial que se ocupa sino la virtud dinámica y activa que corresponde a ese lugar: la de la flecha en el centro del blanco." Vid. Cheng, A.: Historia del pensamiento chino. cit., p. 38 .

21 "La traducción de zhong no deja de resultar problemática y sujeta a malentendidos. A la vez nominal y verbal, el término no indica solo la centralidad espacial que sugiere el sustantivo "medio", sino también una virtud dinámica y activa. Como sustantivo es la vía justa que indica el lugar adecuado y el momento propicio; como verbo, es el movimiento de la flecha que da en el pleno blanco (representado con la grafía 中). Al igual que el arquero que da en el centro de la diana en virtud de la simple precisión de su gesto, que le proporciona su perfecta y natural armonía con el Dao, el zhong es pura eficacia del acto ritual." Cheng, A.: Historia del pensamiento chino. cit., p. 38.-

${ }^{22}$ Además de la dualidad derecho naturalderecho positivo a que antes se aludió, vid. Hesiodo; "Trabajos y días", v 225 y ss. en Obras y Fragmentos, Gredos, Madrid 2006, p. 76 y 77 y Kelsen, H.: "La aparición de la ley de causalidad a partir del principio de retribución.”, en La idea del Derecho Natural y otros ensayos. Editora Nacional. México 1979.

${ }^{23}$ Vid., por ejemplo, Sófocles, Edipo Rey v. 22 y ss. Cátedra, Madrid 2009, p. 204 y también Ullmann, W.: Principios de gobierno y política en la Edad Media, Madrid, Alianza Universidad, 1985, p. 131 y ss. Asimismo puede considerarse al respecto el papel simbólico que aun hoy desempeña la Corona, por ejemplo, en la vigente Constitución española del 78, cuyo art. 56.1 establece: "El Rey es el Jefe del Estado, símbolo de su unidad y permanencia...", mientras que el 56. 3 afirma: "La persona del Rey es inviolable y no está sujeta a responsabilidad..."

${ }^{24}$ Aristóteles, Ética a Nicómaco 1094 a, Centro de Estudios Constitucionales, Madrid 1976 p. 1 y 2 .

${ }^{25}$ Hart, H. LA.: El concepto de Derecho, Editora Nacional, México 1980, p. 110 y 111

${ }^{26}$ Cheng, F.: La escritura poética china cit., p. 29. 
${ }^{27}$ Vid, supra nota 19.

${ }^{28}$ Aristóteles Política 1253 a Instituto de Estudios políticos Madrid 1976, p.4. .

${ }^{29}$ Vid. Welzel, H. Introducción a la Filosofía del Derecho, Aguilar, Madrid 1979, p. 35,

${ }^{30}$ Rousseau, J.J.: El contrato social, Libro I, Capítulo V, en Escritos de Combate, Alfaguara, Madrid 1978, p. 409.

${ }^{31}$ Por decirlo con Constant, B. De la libertad de los antiguos comparada con la de los modernos. Tecnos, Madrid 1988.

32 "Conformando el núcleo del canon confuciano están los "Cinco clásicos" y los "Cuatro libros". Los primeros son los textos que Confucio solía enseñar a sus estudiantes (la palabra china traducida como clásico significa "urdimbre”): el clásico de los cambios Yi Jing, el clásico de los documentos Shu Jing, también conocido como el clásico de la Historia) el clásico de la poesía Shi Jing el registro del Ritual Li Ji y los Anales de primavera y otoño (Chun Jiu). Hay un sexto clásico al que se hace referencia, el clásico de la música (Yue Jing) perdido durante la dinastía Han...

Se considera que los "Cuatro libros" reunidos en un grupo por el especialista neoconfuciano Zhu Xi (1130-1200) concentran las enseñanzas de Confucio. En concreto están formados por las Analectas, Lun yu, una recopilación de dichos y conversaciones del gran sabio chino recogidas por sus discípulos; el Mencio Mengzi, las palabras del célebre seguidor del mismo nombre y el Gran aprendizaje Da xue, así como la Doctrina del medio Zhong yon, cada uno siendo en principio un capítulo del Registro del ritual. El Gran aprendizaje enseña que el primer paso para lograr el orden social es el cultivo y el refinamiento del individuo, y reivindica el ideal del soberano integro desde el punto de vista moral. El tema principal de la doctrina del centro es la interrelación del cosmos y la moral humana, la idea de que los seres humanos y la naturaleza conforman una unidad a través de la sinceridad del esfuerzo. Esos textos fueron las obras centrales de las oposiciones al funcionariado entre los años 1313 y 1905 y al mismo tiempo, constituyen la esencia del neoconfucianismo." Oldstone Moore, J.: "El confucianismo: Orden y virtud." en
Shaughnessy, E. L. (Ed.): China, Blume, Barcelona 2008 , p. 82 y 83 .

${ }^{33}$ Cheng, A.: Historia del pensamiento chino. Barcelona, Bellaterra, 2002, p. 60.

34 "La reforma moral y política, elaborada por Confucio constituye una "educación total", es decir, un método capaz de transformar al individuo ordinario en "hombre superior" (chün$t z u$ ). Cualquiera puede convertirse en un hombre verdadero pero a condición de aprender el comportamiento ceremonial en conformidad con el tao o dicho de otro modo, practicando correctamente los ritos y las costumbres ( $l i$ )." Eliade, M.: Historia de las creencias y las ideas religiosas. Vol. II De Gautama Buda al triunfo del cristianismo. Ediciones Cristiandad. Madrid, 1979, p. 37.

${ }^{35}$ Smith; H.: Las religiones del mundo. Círculo de Lectores, Barcelona 2001, p. 189.

36 “La Gran Enseñanza” IX, en Confucio: Los cuatro libros, cit. p. 310

37 "El tema principal de la doctrina del centro es la interrelación del cosmos y la moral humana, la idea de que los seres humanos y la naturaleza conforman una unidad a través de la sinceridad del esfuerzo." Oldstone Moore, J.: "El confucianismo: Orden y virtud."cit. p. 83.

${ }^{38}$ Buena prueba de ello sería la existencia de la asignatura "Educación para la ciudadanía" lo que a contrario demuestra que el resto de la educación no es para la ciudadanía sino para la vida privada.

${ }^{39}$ Constant, B. De la libertad de los antiguos comparada con la de los modernos. cit., p. 89.

40 “... el peligro de la libertad moderna puede consistir en que absorbiéndonos demasiado en el goce de nuestra independencia privada y en procurar nuestros intereses particulares, no renunciemos con mucha facilidad al derecho de tomar parte en el gobierno político." Constant, B. De la libertad de los antiguos comparada con la de los modernos. Cit. p. 90.

${ }^{41}$ Basta con leer el tenor literal del artículo 87.3 de la vigente Constitución de 1978, esto es: «Una ley orgánica regulará las formas de ejercicio y requisitos de la iniciativa popular para la presentación de proposiciones de ley. En todo caso se exigirán no menos de 500.000 fir- 
mas acreditadas. No procederá dicha iniciativa en materias propias de ley orgánicas, tributarias o de carácter internacional, ni en lo relativo a la prerrogativa de gracia.», para apreciar sus limitaciones: no procede en materias propias de ley orgánica, esto es en las más importantes del ordenamiento jurídico, las que requieren una mayoría cualificada para su aprobación.

${ }^{42}$ Asimismo basta con leer el tenor literal del artículo 92.1 de la vigente Constitución española: «Las decisiones políticas de especial trascendencia podrán ser sometidas a referéndum consultivo de todos los ciudadanos.», para apreciar sus limitaciones: no sólo se trata de un referéndum potestativo, (lit.:"podrán ser sometidas") sino que, además, es meramente consultivo, es decir, no vinculante.

${ }^{43}$ Una de las consecuencias del cambio climático sería precisamente la modificación en los patrones de precipitación. Vid. http://www.treehugger.com/climate-change/three-newreports-this-week-link-extreme-weatherclimate-change.html

${ }^{44} \mathrm{He}$ intentado explicitar esos rasgos en $\mathrm{Re}$ alizando la democracia: 君 人 Individuos y reyes. Eunomía, n. 3, septiembre de 2012-febrero de 2013.p, 125-140. Eunomia.tirant.com. 